\section{VIVIENDA Y ESTRATEGIAS FAMILIARES DE VIDA EN BARRIOS POPULARES CONSOLIDADOS EN BOGOTÁ}

Angélica Patricia Camargo Sierra ${ }^{1}$

\section{Resumen}

Este artículo indaga en la forma en que los hogares propietarios, habitantes de barrios populares consolidados, movilizan su vivienda en sus estrategias familiares de vida. El estudio se realizó en cinco barrios de origen informal de Bogotá, se aplicaron 355 encuestas y 29 entrevistas a propietarios usando el enfoque biográfico. Los resultados dan cuenta de la centralidad económica de la vivienda para los hogares con mayor vulnerabilidad. Se confirma que la casa les ayuda a contener las crisis y en los momentos de bonanza se invierte en ella en primer lugar. En cuanto a las estrategias de movilidad social, la vivienda ha permitido, en algunos casos, apalancar el estudio de los hijos, pero en otros su construcción ha provocado desinversión en capital humano. En síntesis,

\section{HOUSING AND FAMILY LIFE STRATEGIES IN POPULAR NEIGHBORHOODS IN BOGOTÁ}

Abstract

This paper questions the way in which household owners, residents in consolidated workingclass neighborhoods, mobilize their houses in their strategies for family life. This study was conducted in five informal neighborhoods in Bogotá, applying 355 surveys and 29 in-depth interviews using a biographical approach. The results show the economic centrality of housing for these vulnerable households. They confirm that their houses are increasingly important to contain crises and in good times the house is the first to be invested in. In terms of social mobility, in some cases, the house has allowed for children to study, but in others its construction has provoked disinvestment in human capital. In short, for these households, owning a house has a role in containing the insecurities -typical of 
para estos hogares, la casa propia tiene el papel de contener las inseguridades -propias del capitalismo actual y del estado neoliberal- del sistema social.

PALABRAS CLAVE: ESTRATEGIAS DE VIDA; BARRIOS POPULARES; VIVIENDA; INFORMALIDAD URBANA; CRISIS FAMILIARES.

Recibido: 2019-01-09

Aceptado: 2019-07-24 current capitalism and the neoliberal state- of the social system.

\section{KEY WORDS: LIFE STRATEGIES; POPULAR NEIGHBORHOODS; HOUSING; INFORMALITY; HOUSEHOLD CRISIS. \\ URBAN}

Received: 2019-01-09

Accepted: 2019-07-24

1 Colombia. Profesora investigadora de la Escuela de Economía de la Universidad Sergio Arboleda, http://orcid. org/0000-0002-9486-5624. Correo electrónico: angelicapcamargos@yahoo.com 


\section{Introducción}

El rápido crecimiento de las ciudades latinoamericanas durante la segunda mitad del siglo $\mathrm{XX}$, producto de procesos de urbanización informal, ha sido distintivo. En el caso particular de Bogotá, 8700 hectáreas y cerca de 1800 barrios fueron desarrollados informalmente en el transcurso de, por lo menos, setenta años. La urbanización informal constituye una de las principales formas de acceso al suelo urbano y la vivienda para la población de bajos recursos y se define por características específicas. Una de las principales es la calidad irregular del acceso al suelo: generalmente en zonas periféricas, de poco valor inmobiliario y sin dotación de servicios (Camargo y Hurtado, 2013). Las viviendas se producen mediante autoconstrucción en un proceso progresivo que comienza generalmente por una vivienda precaria que pasa por un largo proceso de mejoramiento. Aunque las viviendas mejoran con el tiempo, las condiciones de penuria suelen ser prolongadas, incluso cuando ya están consolidadas (Ward, Jiménez, Di Virgilio y Camargo, 2015). El interés particular de esta investigación se centra en aquellos barrios de origen informal que surgieron en las primeras periferias populares ${ }^{2}$ y están

2 Este estudio se articula con una red de investigación conformada desde 2008 por un equipo de investigadores en América Latina, la cual se interesa por estudiar asentamientos localizados en las primeras periferias urbanas de las ciudades. actualmente consolidados. Específicamente, aquellos asentamientos de origen informal surgidos en Bogotá entre 1955 y 1985.

Aunque en sus comienzos estaban ubicados en zonas periféricas, hoy en día, debido al acelerado crecimiento urbano, la mayoría de estos barrios están ubicados en áreas relativamente cercanas al centro urbano, teniendo todos los servicios públicos, mayor accesibilidad, vías pavimentadas y diferentes rutas y alternativas de transporte público. Los primeros pobladores de estos barrios llegaron, en su mayoría, desde del interior del país, algunos directamente al barrio, y otros a zonas centrales, arrendando vivienda hasta que pudieron obtener el terreno (Camargo, 2014).

Por otra parte, la vivienda es vista como un bien indispensable para la reproducción social, un activo decisivo para los hogares y lo que se busca acumular en primer lugar (Moser, 2009). En particular, para los sectores populares, la vivienda es el proyecto de vida en el que más esfuerzo, dinero y trabajo han invertido (Zaffaroni, 1999). También es uno de los recursos que los hogares suelen movilizar en momentos de crisis y necesidad económica, pues su propiedad los hace menos vulnerables (Bazán, 1999; González de la Rocha, 2009; Moser, 2009). 
Aunque estos hogares han experimentado un mejoramiento general de la situación socioeconómica, sus ingresos son bajos aún. Adicionalmente, los propietarios son ya mayores (en promedio 60 años) lo que implica una reducción de su capacidad productiva. La mayoría han estado vinculados de manera informal al trabajo y carecen de pensión. El ciclo de vida familiar que afrontan estos hogares permite afirmar que se encuentran en situación de vulnerabilidad, por tanto, la vivienda ocupa un lugar central en su condición de vida actual (González de la Rocha, 2009).

Para comprender mejor estas situaciones, este artículo se propone profundizar en el papel económico de la vivienda y la forma en que se moviliza para lograr la sobrevivencia o la mejora del hogar. Se recurre al marco de las estrategias familiares de vida, mediante el cual se analizan aquellos comportamientos y prácticas que emprenden las familias con el fin de mantener y mejorar sus condiciones de vida, su bienestar, su patrimonio, su posición en la estructura de clase y sus condiciones materiales y no materiales de existencia (Bourdieu, 1988; Torrado, 1982). Acciones que emprenden necesariamente desde las opciones, posibilidades y recursos ofrecidos por su contexto doméstico, social y cultural (González de la Rocha, 2009; Wallace,
2002). La adopción de este marco reconoce la capacidad de los individuos o familias para gestionar sus propias vidas y analiza la forma en que movilizan los recursos disponibles. Se profundiza así en las posesiones de los hogares y, en menor medida, en sus carencias (Eguía, 2004; Gutiérrez, 2005). En este caso, se analiza la forma en que movilizan, para su bienestar, el bien más importante que acumulan a lo largo de su vida: la vivienda.

El enfoque de las estrategias familiares de vida (EFV), tiene una larga trayectoria América Latina, aunque también en otros países del mundo (Wallace, 2002). Se ha utilizado principalmente para comprender las estrategias de los hogares ante situaciones difíciles, cambios estructurales y crisis económicas, o en determinadas situaciones de vulnerabilidad. Bajo este marco, el estudio se pregunta: ¿De qué forma los hogares movilizan su vivienda o implementan determinadas prácticas residenciales en sus estrategias familiares de vida? En particular, se analizaron tres tipos de estrategias: aquellas orientadas a mantener su bienestar presente, las que se dirigen a afrontar determinados momentos de crisis y de bonanza, y, finalmente, las estrategias para mejorar sus condiciones de bienestar futuro (propios o de la siguiente generación), es decir, de movilidad social. 


\section{El enfoque de las estrategias familiares de vida (EFV)}

Como otros enfoques en las ciencias sociales, el de las EFV ha estado en permanente mutación y transformación. Ha pasado por múltiples denominaciones, diferentes comprobaciones empíricas, críticas y redefiniciones.

Inicialmente surge bajo el nombre de estrategias de sobrevivencia y tiene su origen hacia los años sesenta y setenta (Duque y Pastrana, 1973). Surgió como una forma de responder al enfoque estructuralista, que no reconocía la capacidad de agencia de familias o individuos (González de la Rocha, 2009). El interés inicial ha sido estudiar las estrategias implementadas por las familias de sectores populares para afrontar la pobreza, las crisis y las transformaciones económicas, principalmente aquellas provocadas por políticas como el ajuste estructural y la precarización laboral en el caso de América Latina (Arteaga, 2007). También se analiza la forma en que se optimizan los recursos disponibles para incrementar su bienestar.

Para argumentar la relevancia de este enfoque, en las ciencias sociales en general y en los estudios sobre la vivienda en particular, es preciso verlo desde las tres perspectivas que propone Wallace (2002): como concepto, como método y como unidad de análisis. Como concepto, tiene en cuenta las motivaciones y la capacidad de agencia de los actores en la sociedad, y su relevancia radica en comprender los efectos sobre la economía de los hogares que imponen los constantes cambios inherentes al proceso de globalización y a las economías posfordistas. Como método de análisis, busca un punto de intersección entre las estructuras sociales y el comportamiento y las decisiones de los hogares. Por lo tanto, su aplicación es relevante en contextos donde es cada vez más importante la economía informal y donde la inestabilidad obliga a los hogares a desplegar estrategias de ajuste, adaptación y sobrevivencia; de hecho, las economías informales suelen estar estrechamente relacionadas con los procesos de urbanización informal (Álvarez, 2009). El hogar como unidad de análisis, propio de este enfoque, cobra importancia en los estudios sobre la vivienda. En tanto son categorías indisociables (hogar-vivienda), comprender mejor los cambios en las prácticas sociales y económicas de los hogares tiene implicaciones para el diseño de la política habitacional y urbana.

Las estrategias de vida refieren a determinados arreglos, prácticas, comportamientos o acciones que se implementan en el ámbito del hogar con el fin de garantizar la sobrevivencia, la reproducción de la vida y de la fuerza de trabajo o, simplemente, mejorar las condiciones de bienestar. En términos analíticos, el enfoque busca describir estas prácticas e intentar explicarlas o 
comprenderlas (Arteaga, 2007; Barabino, Bocero, Prandín y Rosenthal, s.f.; Eguía, 2004). Tales estrategias solo pueden ser construidas por sus actores en función de las opciones y posibilidades que les ofrece el contexto social, cultural, familiar y espacial en el que están inmersos (González de la Rocha, 2009). Se les ha dado diversas denominaciones: estrategias de sobrevivencia (González de la Rocha, 1986); estrategias de adaptación (Moen y Wethington, 1992); estrategias de supervivencia (Duque y Pastrana, 1973); estrategias de existencia (Sáenz y Di Paula, 1981); estrategias de reproducción social (Bourdieu, 2011); y estrategias de vida (Torrado, 1982). Aunque no todas aluden exactamente a lo mismo, recogen en términos generales los mismos propósitos analíticos.

Torrado (1982), adoptó el término de estrategias familiares de vida (EFV) después de notar que las anteriores nociones solo serían válidas para estudiar la reproducción económica de los hogares pobres, no resultando útiles para estudiar otros ámbitos de la reproducción (no económicos; por ejemplo, biológicos) en otros grupos y clases sociales. Las define como:

Aquellos comportamientos de los agentes sociales de una sociedad dada que, estando condicionados por su posición social -(o sea por su pertenencia a determinada clase o estrato social)- se relacionan con la constitución y mantenimiento de unidades familiares, en el seno de las cuales pueden asegurar su reproducción biológica, preservar la vida y desarrollar todas aquellas prácticas económicas y no económicas indispensables para la optimización de las condiciones materiales y no materiales de existencia de la unidad y de cada uno de sus miembros (Torrado, 1982, p. 4).

Partiendo de que la vivienda es un recurso importante en el despliegue de las estrategias de vida, resulta útil la propuesta conceptual de Moser (2009), sobre vulnerabilidad y acumulación de activos. En este marco, la vulnerabilidad refiere a la inseguridad en el bienestar de los hogares o individuos, siendo causada por los cambios en el entorno y asociada a baja capacidad de respuesta y poca resiliencia. En la reducción de la vulnerabilidad de los hogares, los activos son vistos como medios importantes de resistencia. Así, en la cartera de activos que gestionan los hogares, la vivienda ocupa un papel central (Moser, 2009; Ortiz y Diaz, 2018).

Bajo el enfoque de las EFV, tres aspectos requieren especial atención en esta investigación: i) la noción de estrategia; ii) la articulación de las estrategias con las estructuras sociales; y iii) los objetivos que se persiguen. 


\section{LA NOCIÓN DE ESTRATEGIA: ENTRE LA RACIONALIDAD Y EL SENTIDO PRÁCTICO}

Un de los aspectos de este enfoque que ha generado más discusión es la noción de estrategia y el nivel de racionalidad o conciencia de los agentes que las implementan. Katzman (1999) define las estrategias como "formas particulares de articulación de recursos para el logro de una meta" (p. 32). Esta noción surge en las ciencias sociales, según Bourdieu y Lamaison (1985), como el "instrumento de una ruptura con el punto de vista objetivista y con la acción sin agente que supone el estructuralismo" (p. 2).

Para Bourdieu (1989), las estrategias no son necesariamente el resultado de un ejercicio consciente y racional - aunque tampoco necesariamente irracional- de estructuración de medios y fines, sino el producto del sentido práctico. La racionalidad de los hogares es, en cualquier caso, histórica y socialmente construida y por lo tanto enmascara e involucra situaciones de clase, por lo que muchas veces se trata más bien de respuestas adaptativas forzosas (Barabino et al., s.f.). Para Garrido y Gil (1996) los resultados (incluso lo más estratégicos) de las acciones de los hogares son más bien colaterales y las intenciones muchas veces resultan contradictorias e inciertas. De modo que algunas estrategias pueden tener un ejercicio de cálculo detrás, mientras otras pueden ser solo reacciones del hogar ante situaciones específicas, y en otros casos son solamente adaptaciones a situaciones complejas (Barabino et al., s.f.; Katzman, 1999).

De cualquier forma, el enfoque visibiliza la capacidad de agencia de los hogares y reconoce que los actores tienen la posibilidad de reflexionar y modificar sus prácticas, de tomar decisiones y de elegir opciones y cursos de acción en su vida (Giddens, 1995; Moser, 2009).

\section{ENTRE LOS ASPECTOS ESTRUCTURALES Y LAS PARTICULARIDADES DE LOS HOGARES}

Aunque este enfoque reconoce la gran capacidad de los sectores populares para desplegar estrategias de maneras creativas y recursivas, esto no implica que sean ilimitadas y que operen en cualquier tipo de condiciones (González de la Rocha, 2009). Por lo tanto, se debe tener presente la forma en que operan los constreñimientos del contexto social y cultural, la posición de los hogares en la estructura social y cuestiones estructurales como las condiciones macroeconómicas y el mercado laboral y residencial (Di Virgilio, 2011). De acuerdo con Torrado (1982), el hogar es una instancia mediadora que hace efectiva la influencia de la estructura social en sus comportamientos demográficos, económicos y sociales.

La mayoría de las estrategias se plantean y se gestan al nivel del hogar; sin embargo, esto no implica la ausencia de planes individuales ( $\mathrm{Di}$ 
Virgilio, 2011). Además, las condiciones económicas y de vulnerabilidad del hogar están estrechamente ligadas con algunas características específicas, como: el tamaño, la cantidad de miembros que trabajan y el ciclo que atraviesa el hogar. González de la Rocha (2009) identifica tres ciclos o fases en la evolución de los hogares. La fase de expansión, que comienza con la constitución de la pareja y finaliza cuando concluye la edad fértil de la mujer; la fase de consolidación, en la cual el hogar ha terminado su período de reproducción y al menos uno de los hijos trabaja; y la fase de dispersión, que comienza cuando los hijos abandonan el hogar paterno. En esta última, el núcleo que permanece suele estar en la vejez o aproximándose a ella. Específicamente, los sectores de más bajos recursos presentan mayor vulnerabilidad en la primera y la tercera fase, por lo que deben realizar ajustes en sus estrategias de vida (González de la Rocha, 2009).

\section{TIPOS DE ESTRATEGIAS: OBJETIVOS QUE PERSIGUEN Y RECURSOS OUE MOVILIZAN}

Buena parte de los hallazgos en este enfoque apuntan a describir los tipos de estrategias más comunes implementadas por los hogares en función de sus proyectos de bienestar. Para precisar los tipos de estrategias, se tienen en cuenta por lo menos dos elementos: los recursos que movilizan (trabajo, activos, bienes, redes, etc.) y los objetivos que se persiguen en función del horizonte temporal estratégico.
Cornia (1987) identifica estrategias orientadas a generar más recursos y a proteger el nivel de ingresos (o aumentarlo) y estrategias orientadas a optimizar los recursos existentes. Las primeras pueden concretarse a través del incremento del trabajo asalariado del hogar, o mediante autoempleo, endeudamiento, venta de bienes, búsqueda de apoyos externos o migración. Las segundas refieren a modificar los niveles de consumo, desinvertir en capital humano o a la reorganización familiar. De acuerdo con González de la Rocha (2009) hay estrategias de reproducción, orientadas a asegurar el bienestar de los miembros del grupo en el mediano y largo plazo y estrategias de sobrevivencia o enfrentamiento, para enfrentar situaciones difíciles de la vida diaria.

En el análisis de las EFV, y en particular para este estudio, resulta muy importante comprender los recursos o activos con los que cuentan las familias, su capacidad para movilizarlos y la forma en que los movilizan. Este aspecto rompe con los análisis tradicionales de medición de la pobreza, pues, en su enfoque, no resulta tan importante saber qué carencias tienen, como sí saber qué poseen y la forma en que lo movilizan o agencian para mejorar sus condiciones o para lograr determinado objetivo (Gutiérrez, 2005). Los recursos que suelen movilizar los sectores populares en momentos de crisis son: la fuerza de trabajo del hogar, los recursos productivos (entre ellos la vivienda) y las redes familiares y 
sociales (Eguía, 2004; Hintze, 2004; Katzman, 1999; Moser, 2009; Vallejos y Leotta, 2013; Zaffaroni, 1999).

En virtud de la articulación entre los propósitos y los recursos que se movilizan, resulta útil para la presente investigación la clasificación de Katzman (1999), que además recoge las diferentes clasificaciones antes mencionadas. En este texto se propone analizar la forma en que los hogares movilizan la vivienda en función de tres tipos de estrategias: i) estrategias para mantener (o evitar que se deterioren) las condiciones de bienestar presentes; ii) estrategias para afrontar choques económicos (crisis y bonanzas); iii) estrategias para mejorar la situación de bienestar futuro, es decir de movilidad social.

\section{Aspectos metodológicos}

Para el diseño metodológico de la investigación, se adoptó el enfoque biográfico, caracterizado por el uso y análisis sistemático de documentos vitales como autobiografías, historias o relatos de vida, crónicas, cuestionarios biográficos, etc. (Sautú, 1999). Se recurre a este enfoque pues las estrategias de vida deben analizarse en un marco de análisis temporal y contextual amplio, que implica comprender el curso de vida del hogar en relación con el contexto social, cultural y, en este caso, urbano en el que se inscriben las unidades de análisis. Este enfoque permite avanzar en la comprensión de los tiempos de vida de las personas y sus contextos. Reconoce que el curso de vida de los individuos está embebido y formado por los tiempos históricos y los lugares que se experimentan a lo largo de la vida (Elder, 2000).

Para esta investigación se aplicaron 355 encuestas biográficas a propietarios en cinco barrios populares consolidados en Bogotá. Se realizó una muestra probabilística con tamaño máximo, con nivel de confianza establecido de (95\%) y porcentaje de error del 5\%. Las unidades de muestreo fueron lotes y la escogencia se realizó de manera aleatoria-sistemática. Las encuestas se realizaron entre marzo y abril de 2015. Se utilizó una estrategia de múltiples casos que, como conjunto, pudiera dar cuenta del universo de estudio (Stake, 1994). Adicionalmente, se realizaron 29 entrevistas a profundidad con relatos de vida a una submuestra de los propietarios encuestados (Tabla 1). Para la escogencia de los entrevistados se utilizó la metodología de identificación de "casos interesantes" (Ward, Jiménez y Di Virgilio, 2014). La inclusión de los entrevistados tuvo en cuenta criterios asociados a las articulaciones entre las trayectorias de vida y de la vivienda, las tipologías y características del hogar y de la vivienda. Las entrevistas se realizaron entre abril y junio de 2016.

En los cuestionarios de la encuesta se abordaron, entre otros temas: las características de la edificación y la vivienda, la historia de la propiedad, las características de los miembros del 
hogar, las trayectorias de vida del propietario, las actividades económicas en la vivienda, el afrontamiento de crisis y bonanzas económicas y las percepciones sobre la condición social y económica.

En las entrevistas a profundidad se realizaron relatos de vida, orientados a reconstruir trayectorias residenciales, sociales y laborales. Se profundizó en la conformación del hogar, la llegada al barrio y la construcción progresiva de la casa. Se abordó el papel de la vivienda en diferentes aspectos de la vida del hogar, el uso y la ocupación de los diferentes espacios de la casa y sus cambios en el tiempo. Se identificaron y caracterizaron las diferentes formas de aprovechamiento económico y productivo que ha tenido la vivienda. Se ahondó en el análisis de los momentos de crisis y bonanza, en sus causas, consecuencias y prácticas. Se abordaron los proyectos y aspiraciones de los miembros del hogar y las acciones que han puesto o planean poner en práctica para conseguirlos. Se indagó también sobre los sentidos que les daban a sus estrategias de vida.

Se utilizó una estrategia de análisis mixta (con datos cuantitativos y cualitativos) mediante criterios de complementación y triangulación (Bericat, 1998). Se buscó la convergencia de los resultados de las fuentes de datos para responder las preguntas de la investigación. Para el procesamiento de las entrevistas se empleó una técnica de análisis del discurso y para las encuestas se utilizaron técnicas estadísticas de análisis descriptivo.

El universo de estudio está constituido por el conjunto de barrios populares consolidados en Bogotá, que surgieron entre 1955 y 1985. En total se identificaron 1860 barrios de origen informal en Bogotá (ver Figura 1 para su localización), de los cuales 1243 surgieron en el período indicado. Estos últimos suman cerca de 244.000 lotes y ocupan 6062 hectáreas y aproximadamente 925.000 habitantes.

Se analizaron cinco barrios, de origen informal, en Bogotá: San Martín de Porres, Atenas, El anhelo, Japón e Ismael Perdomo. San Martín de Porres es uno de los más antiguos y surgió del cierre de una cantera cuyos dueños dejaron la tierra a los trabajadores como forma de pago, para devenir luego en una toma organizada. Perdomo y Atenas resultaron de parcelaciones agenciadas por propietarios de tierra que no cumplían las normas urbanas de la época. Los más recientes, Japón y El anhelo surgieron por parcelaciones promovidas por urbanizadores ilegales. En todos los casos hubo procesos de gestión colectiva de las dotaciones urbanas y de la producción de las viviendas mediante autoconstrucción (Tabla 2). 
TABLA 1. ENTREVISTAS Y ENCUESTAS POR BARRIO.

\begin{tabular}{|lll|}
\hline Barrio & Entrevistas & Encuestas \\
\hline Atenas & 5 & 64 \\
\hline El Anhelo & 6 & 87 \\
\hline Japón & 5 & 55 \\
\hline Perdomo & 7 & 110 \\
\hline San Martin de Porres & 6 & 39 \\
\hline Total general & 29 & 355 \\
\hline
\end{tabular}

Fuente: elaboración propia

TABLA 2. DATOS DE LOS BARRIOS.

\begin{tabular}{|lllllll|}
\hline Nombre & Localidad & $\begin{array}{l}\text { Año de } \\
\text { origen }\end{array}$ & Hab/ha & No. Lotes & Área (has) & Pob \\
\hline Ismael Perdomo & Ciudad Bolívar & 1976 & 388 & 164 & 2,3 & 886 \\
\hline Atenas & San Cristóbal & 1955 & 267 & 149 & 3,0 & 805 \\
\hline San Martin de Porres & Chapinero & 1960 & 134 & 133 & 4,7 & 637 \\
\hline El anhelo & Bosa & 1987 & 483 & 955 & 10,7 & 5157 \\
\hline Japón & Suba & 1973 & 303 & 409 & 7,3 & 2209
\end{tabular}

Fuente: elaboración propia con datos de la SDP. 
FIGURA 1. LOCALIZACIÓN DE LOS BARRIOS.

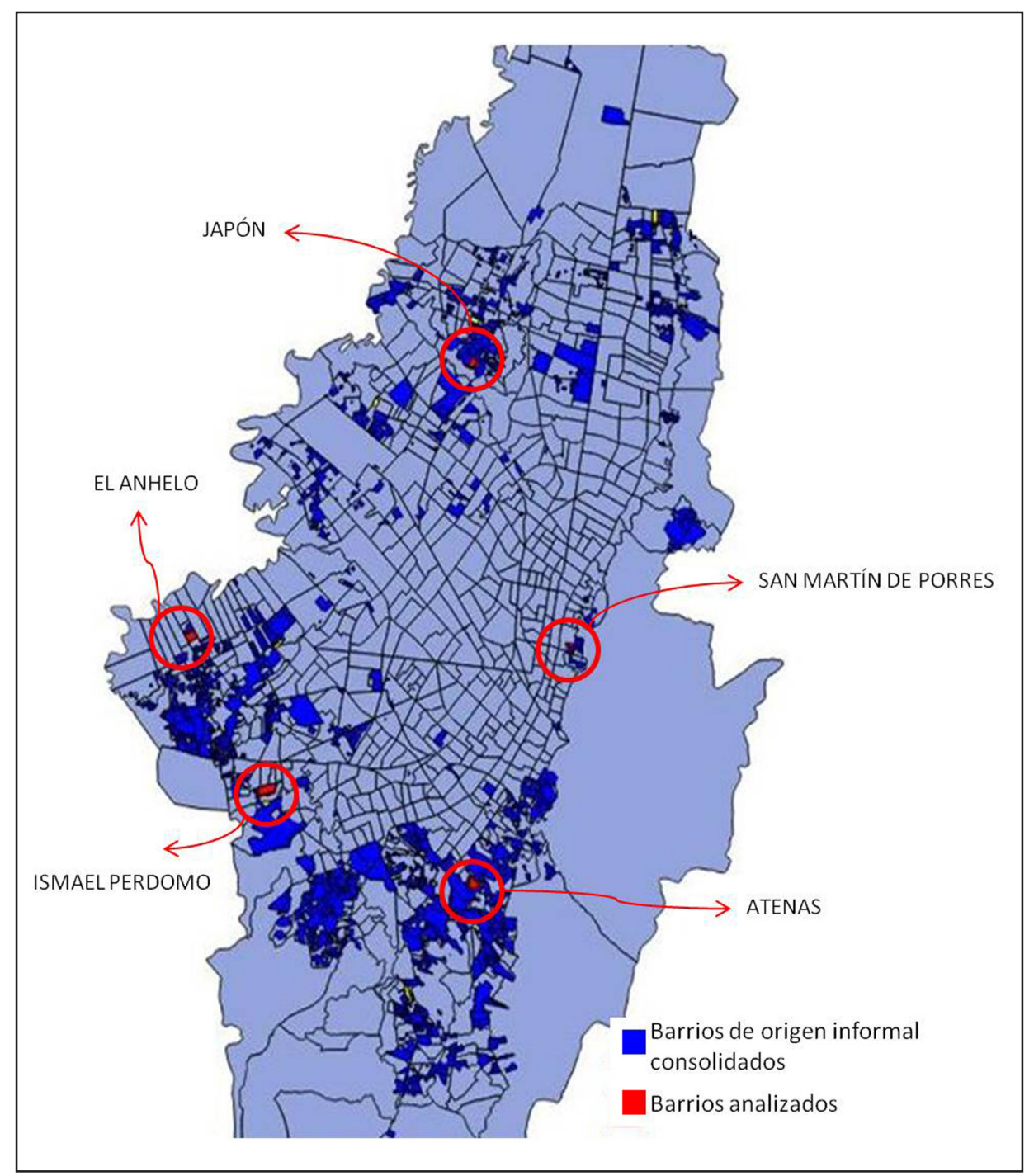

Fuente: elaboración propia con datos de la Secretaría Distrital de Planeación de Bogotá (SDP). 


\section{La vivienda en las estrategias de vida}

\section{CARACTERÍSTICAS DE VIVIENDAS Y HOGARES}

Las viviendas son generalmente grandes y subdividas. Aunque el área de terreno promedio es de $120 \mathrm{~m}^{2}$, en Perdomo es de casi $180 \mathrm{~m}^{2}$ y en El anhelo de 72 (que es la moda para todos los casos). En Atenas y San Martín de Porres los lotes se acercan a los 115 (Tabla 3). Los propietarios calculan que sus viviendas tienen cerca de $150 \mathrm{~m}^{2}$ construidos.

A pesar de que cada unidad es un solo predio y un solo propietario, las casas suelen estar subdivididas. Hay en promedio 2,2 viviendas y 7,5 personas por edificación. La forma de subdivisión común es construir en cada piso uno o dos apartamentos. Las edificaciones tienen entre uno (22\%), dos (50\%) y tres pisos (24\%). Cada piso suele tener dos alcobas, una sala y/o comedor, un baño y una cocina. El 32,5\% de las casas no tiene patio ni terraza y el 70\% tampoco garaje. El 31,6\% cuenta con un local, por lo menos. El $71 \%$ de los encuestados considera que su casa aún no está terminada y, de estos, el 51\% quiere construir más.

Las características del hogar son determinantes para analizar y comprender las estrategias económicas de los hogares. Varios estudios han advertido las relaciones entre la vulnerabilidad del hogar y su composición, tamaño y vinculación con el mercado de trabajo (González de la Rocha, 2009; Moser, 2009; Perlman, 2010).

El tamaño promedio de los hogares es de cuatro personas, mayor que el promedio de la ciudad (que es 3,2 según la encuesta multipropósito 2014). El barrio San Martín de Porres tiene los hogares más grandes $(4,4)$ y Atenas y Perdomo los más pequeños $(3,8)$.

Al analizar la conformación de los hogares se pueden identificar tres realidades: i) abuelos viviendo solos (5,2\% de los hogares) por haber enviudado, por haberse separado o por no haberse casado; ii) hogares en fase de dispersión y de nido vacío, donde la edad promedio de los padres $(59,4$ años) e hijos $(25,9)$ indica que los hijos están empezando a formar sus propios hogares y a dejar el hogar paterno; iii) hogares extensos (35,2\%), que superan ampliamente el promedio de la ciudad (21,5\%), cuyo tamaño es de 5,2 personas y donde hay por lo menos un nieto en el 76,4\% de ellos. Según González de la Rocha (2009), conformaciones familiares como éstas conllevan mayor vulnerabilidad. Aunque en los hogares extensos suele aumentar la fuerza de trabajo joven, también aumenta el número de niños (nietos), lo que genera mayor desequilibrio económico. 
TABLA 3. CARACTERÍSTICAS PROMEDIO.

\begin{tabular}{lllllll} 
Barrio & $\begin{array}{l}\text { Edad del } \\
\text { propietario }\end{array}$ & $\begin{array}{l}\text { Tamaño del } \\
\text { lote }\end{array}$ & $\begin{array}{l}\mathrm{M}^{2} \\
\text { construidos }\end{array}$ & $\begin{array}{l}\text { Personas por } \\
\text { vivienda }\end{array}$ & $\begin{array}{l}\text { Hogares por } \\
\text { vivienda }\end{array}$ & $\begin{array}{l}\text { Personas por } \\
\text { hogar }\end{array}$ \\
\hline Atenas & 59 & 118,1 & 164,3 & 7,3 & 2,3 & 3,8 \\
El Anhelo & 51,4 & 66,8 & 122,8 & 7,3 & 2,0 & 4,1 \\
Japón & 59,3 & 84,3 & 181,6 & 8,7 & 2,6 & 3,9 \\
Perdomo & 66,1 & 180,3 & 219,5 & 7,3 & 2,2 & 3,7 \\
$\begin{array}{l}\text { San Martin de } \\
\text { Porres }\end{array}$ & 56,6 & 109,4 & 157,9 & 7,9 & 2,4 & 4,2 \\
Total & 58,7 & 119,1 & 156,2 & 7,6 & 2,3 & 3,9
\end{tabular}

Fuente: elaboración propia

\section{ESTRATEGIAS PARA MANTENER SU BIENESTAR PRESENTE: LA VIVIENDA EN LA ECONOMÍA DE LAS FAMILIAS}

Diversos estudios han dado cuenta de la importancia económica que tiene la vivienda en los sectores populares y en particular la posibilidad de utilizarla para obtener recursos adicionales (Peattie, 1974). Tal como se evidencia en las características de los hogares, los propietarios y jefes de estos son ya mayores, muchos de ellos no trabajan, no tienen pensión y tienen bajos ingresos, de modo que su vivienda se convierte en un recurso muy importante para mantener sus condiciones de bienestar. Para comprender el papel de la vivienda en la economía actual de estos hogares, se indagó principalmente por el uso económico que le dan a su casa, el destino de estos recursos y la importancia en sus ingresos.

La mayoría de los encuestados (41,7\%) recibe entre uno y dos salarios mínimos, y el 24\% menos de uno. El 66,8\% saca algún provecho económico de la vivienda. La práctica más común es alquilar espacios de la casa (Jiménez y Camargo, 2015). Otra práctica común es tener un negocio (atendido por el propietario) o desarrollar una actividad laboral en la vivienda (taller de costura, carpintería, etc.). En estos últimos dos casos la vivienda constituye un medio importante para la obtención del ingreso, sumado a lo que Aulestia (2009) denomina la renta imputable, que es el dinero que obtendría el propietario si decide alquilar el espacio que ocupa. 
El 37,7\% arrienda algún apartamento. Esta práctica es más común en El Anhelo (50\%) (el barrio más reciente) y menos en Perdomo y San Martín de Porres (cerca del 25\% y en los más antiguos). Se arriendan dos apartamentos en cada edificación y el canon promedio es de \$122 dólares, siendo menor la renta en el barrio Atenas (\$110 dólares) y mayor en San Martín de Porres (\$142 dólares), que tiene la localización más central de los barrios estudiados. Se arriendan cuartos en el 5,5\% de los casos. Suelen arrendarse entre uno y tres cuartos de la vivienda y el canon promedio es de $\$ 82$ dólares. Algunos propietarios $(8,1 \%)$ arriendan locales, principalmente en Perdomo (17,5\%) cuya vocación comercial es mayor. En pocos casos tienen arrendada la totalidad de la casa; normalmente solo lo hacen por apartamentos o cuartos.

En el 13,7\% de los casos algún miembro de la familia utiliza la casa para trabajar, principalmente con negocios propios (tiendas, panaderías, etc.), talleres (carpintería, costura, ornamentación, etc.) y cuidado de niños. Si bien esto suele ser mayoritario para las mujeres (44,2\%), en este estudio resulta también una opción importante para los hombres (36\%). En el 19,5\% de los casos ambos miembros de la pareja trabajan en la misma vivienda.

En las entrevistas se profundizó en estas prácticas y en el destino que se da a los recursos obtenidos con la vivienda. Son diversas las alternativas de uso de los recursos que obtienen; en muchos casos se utilizan en los gastos generales de la familia o en el mantenimiento de la vivienda. Es común que los ingresos que reciban se inviertan en ampliar la vivienda, lo que a futuro les permite producir mayores ingresos mediante la casa. Uno de los casos que ejemplifica esto es el de María Elisa del barrio San Martín de Porres. María Elisa, de 63 años, fue a vivir con su esposo en la vivienda heredada de su madre, que adecuaron y ampliaron para vivir, arrendar y montar un negocio. La señora ha logrado construir prácticamente toda su casa con lo que le da el negocio y la renta de espacios. Su relato lo explica muy bien:

Monté mi negocio y empecé a terminar toda la casa, luego construí allá y empecé a arrendar esas tres piezas, y del arriendo y del tejo fui terminando de construir la casa... lo que da de allí se mete allá y lo que da de acá se mete allá (María Elisa, entrevista personal, 14 de mayo, 2016).

En algunos casos, los recursos obtenidos por la vivienda constituyen el principal, e incluso, el único ingreso del hogar. Luis Enrique, del barrio Atenas y cuyo caso se ampliará más adelante recibe la totalidad de sus ingresos del aprovechamiento económico de su vivienda. Lucio, también en Atenas, llegó hace 30 años al barrio, desde ese momento instaló una panadería en la casa en la que trabaja con su esposa, y junto con el arriendo de un apartamento constituye la totalidad del ingreso familiar. 
El ingreso proveniente de la vivienda es particularmente importante para las mujeres jefes de hogar. Blanca, por ejemplo, vive en el barrio Atenas y es separada desde hace 20 años. Actualmente vive con dos hijos y dos nietos. Atiende sábados y domingos un negocio de venta de cerveza y arrienda el tercer piso de la casa: "yo vivo de lo que me da esta vivienda, pero pues no me gusta pedirle a los hijos, ellos sí me ayudan pero también tienen sus gastos" (Blanca, entrevista personal, 5 de mayo, 2016). Nuevamente en el caso de María Elisa, jefe de hogar desde que su esposo enfermó, todos sus ingresos provienen de la casa y no solamente los usa para cubrir los gastos de la casa, sino que también para ayudar a sus nietos.

Mis ingresos son de la casa. Con lo que cojo pago luz, agua, gas y todo con lo de la casa. También le pago el estudio a mi niño menor, con lo de la casa, y también les ayudo a mis nietos. Abuelita que para las onces, que para libros, que un par de zapatos, todo, todo lo que me pidan yo les ayudo (María Elisa, entrevista personal, 14 de mayo, 2016).

Los ingresos que logran obtener en su vivienda, les ayudan a contener la vulnerabilidad propia de su ciclo de vida.

\section{LA VIVIENDA EN MOMENTOS DE CRISIS Y BONANZAS}

Las familias y las personas experimentan a lo largo de sus vidas momentos de choques económicos. Estos pueden ser negativos (crisis) o positivos (bonanzas). Las causas de crisis económicas en los hogares son, en general, la muerte de un familiar, la desvinculación laboral, la enfermedad, entre otras (Moser, 1996).

Cuando los ingresos disminuyen los hogares emprenden estrategias para aumentar el ingreso o reducir el gasto (Moser, 1996). Para aumentar los ingresos lo principal es ampliar la vinculación del hogar al mercado laboral. Esto puede ser mediante la entrada de nuevos miembros, el aumento de las horas laborales, la vinculación al mercado informal u otra forma desventajosa de vinculación laboral, la venta de bienes, el endeudamiento, entre otras (González de la Rocha, 1986; Zaffaroni, 1999). La diversificación de ingresos es una herramienta fundamental para afrontar los choques económicos, y el uso de la vivienda como parte de esto contribuye a disminuir la vulnerabilidad ante las crisis (Bazán, 1999; Moser, 2009). En cuanto a la disminución de gastos, además del sacrificio en consumo de alimento, vestuario, salud y otros bienes, se cuenta la desinversión en capital humano (Núñez, 2012; Prada, 2006). 
Entre la población estudiada, el 56\% afirmó haber pasado por un momento de crisis económica. La principal causa fue la pérdida del empleo (47,8\%), en segundo lugar, un mal negocio $(17,1 \%)$ y en tercero, cuestiones de salud (13,3\%) o la muerte de algún familiar (7,9\%). En general, les resultó difícil identificar momentos específicos de crisis económica: "yo he estado en crisis toda la vida" fue una respuesta común.

Entre las principales estrategias para afrontar los problemas económicos está incrementar el tiempo de trabajo (25,9\%), diversificar los ingresos $(25,6 \%)$ o restringir el consumo $(38,7 \%)$. También pedir dinero prestado y el uso de los ahorros. Las estrategias residenciales las mencionó el 7,1\%. La principal consiste en arrendar alguna parte de la vivienda y luego instalar un negocio en la misma (5\%).

En las entrevistas se indagó con mayor profundidad por el uso de la vivienda en estos momentos. Uno de los casos fue el de Luis Enrique, quien llegó del Huila, desplazado por la violencia, a casa de su hermana en el barrio Atenas, quien la tenía desocupada para venderla. Al principio le fue muy difícil adaptarse porque en el campo se dedicaba solo a cultivar la tierra. Sin embargo, su esposa decidió preparar tamales para venderlos cada domingo. Tienen una pequeña venta de artículos para bebé y junto a su hija sacan a la puerta, todas las tardes, un puesto de venta de arepas y además arriendan un cuarto (entrevista personal, 6 de mayo, 2016).

Otro caso representativo es el de Fátima, de 54 años que llegó a vivir al barrio Atenas hace 20 años. Cuando su esposo se enfermó, decidió pedir el local que tenían arrendado y montar su propio negocio: "Llevamos dos años con el negocio. Cuando mi esposo se empezó a enfermar, pedimos el local y lo arreglamos. Pues no se vende en cantidades, pero bien, vivimos del negocio y los arriendos" (Fátima, entrevista personal, 26 de mayo, 2016).

También el de Mélida, quien ha vivido toda su vida en el barrio Perdomo, primero en la casa de su familia, después de casarse, en casa de sus suegros y luego en la vivienda que construyeron en el barrio. Decidieron instalar una ferretería en la casa cuando la salud de su esposo no le permitió trabajar más en la construcción. Desde que su esposo murió, se dedica a atender la misma ferretería para afrontar las deudas que dejó.

Cuando falleció mi esposo, eso quedaron muchas deudas y yo me enfermé, eso me dio un patatús. El médico me dijo, sin yo contarle nada: a usted le pasa algo y está muy estresada y tiene que calmarse, si debe plata, hable con los proveedores. Hice lo que me dijo y los proveedores me dejaron pagarles de a poquito. Ya pude salir de todas esas deudas (Mélida, entrevista personal, 30 de abril, 2016). 
La vivienda no solamente soporta en momentos de crisis al propietario, sino también a otros parientes. Generalmente los hijos, cuando deciden formar un hogar y prefieren -o cuando no tienen otra opción- vivir en casa de sus padres. La práctica de compartir la edificación es realizada por el $64,8 \%$ de los propietarios. Entre estos, el 34,2\% la comparte solo con familiares.

Se analizaron también las bonanzas económicas, dado que se conoce mucho sobre el manejo de las crisis, pero poco sobre cómo se administran los momentos de bonanza o mejora económica. Esto podría ser útil para entenderlos, potenciarlos y hacerlos más sostenibles en el tiempo. Según Núñez (2012), estas bonanzas se generan usualmente cuando mejora la situación laboral, cuando los mayores ingresos son permanentes, permitiendo un proceso de movilidad ascendente. En su investigación, la vivienda fue identificada como el factor material más importante de movilidad social desde la percepción de los hogares.

En la encuesta se preguntó si los propietarios han experimentado momentos de bonanza o mejora económica, las causas de éstas y las opciones que toman cuando tiene recursos extra. Se buscó identificar si hay prácticas residenciales que promuevan una bonanza económica, y si experimentarla provoca alguna práctica residencial en particular, como mejorar o ampliar la vivienda, comprar otra vivienda o mudarse.
El 34\% de los encuestados afirmó haber pasado por un momento de bonanza económica. Entre sus causas están: el éxito en un negocio (30,4\%); el conseguir un mejor trabajo (25\%), alguna ganancia ocasional (17\%) o el aporte de otros miembros del hogar (11\%). La mayoría $(73,2 \%)$ dice que esta bonanza continúa hasta ahora. En cuanto al uso del dinero adicional, las opciones más mencionadas fueron en este orden: ampliar o mejorar la vivienda (29,2\%), ampliar los gastos $(24,7 \%)$, salir de vacaciones (19,7\%), ahorrar (11,7\%), comprar bienes $(8,6 \%)$ e invertir en educación (8,3\%).

En las entrevistas se profundizó en estos momentos de bonanza y en el papel de la vivienda. Se encontró el caso de Fabiola en Bosa, quien ganó un pequeño premio de lotería y decidió invertirlo en la construcción de un tercer piso y en la compra de electrodomésticos (entrevista personal, 23 de mayo de 2016).

Nancy construyó gran parte de su casa gracias al éxito de su negocio de cerveza, que por cierto empezó luego de una quiebra de su esposo:

Cuando el cuchito [su esposo] quebró, entonces yo monté un depósito de cerveza cerca de Corabastos, empecé con un millón quinientos que había ahorrado de los arriendos, y empecé con 30 cajitas de cerveza. Y empezaron los clientes a pedir y a pedir. $Y$ trabajé en eso como año y 
medio. Con eso le metí como 25 millones de pesos en este cuarto piso. (Nancy, entrevista personal, 24 de mayo, 2016)

Aunque la mayoría de los entrevistados no lograron identificar momentos de especial bonanza, sienten que sus condiciones económicas han mejorado y que su casa es un reflejo de ello. La inversión en la vivienda en momentos de mejora fue contundente y da cuenta de la importancia económica de ésta en su vida, en sus estrategias y aspiraciones.

\section{ESTRATEGIAS DE MOVILIDAD SOCIAL: PENSIÓN DE VEJEZ Y EDUCACIÓN PARA LOS HIJOS}

Según Katzman (1999), las estrategias de movilidad social son acciones, aspiraciones y proyectos de la familia orientados a mejorar el bienestar futuro, lo que implica no solo al individuo sino también a su hogar y (sobretodo) a sus hijos. Sobre la base de algunos estudios previos (Gaviria, 2005; Perlman, 2010), en la encuesta se hicieron preguntas para valorar la percepción sobre su movilidad social y los factores que la promueven.

Un primer aspecto tiene que ver con su nivel de satisfacción con la vida. Aunque la mayoría está muy satisfecha con su vida $(74,3 \%)$, lo están mayormente con su vivienda (94\%). La mayoría piensa que su situación económica es mejor que la de sus padres (81\%) y que cuando conformaron su hogar (73,3\%). También afirman que la vida de sus hijos ha sido mucho mejor que la de ellos $(85,5 \%)$.

En cuanto a los aspectos que consideran más importantes para mejorar en la vida, el más mencionado fue: la fe en Dios $(59,1 \%)$, seguido del estudio (57\%), el trabajo duro $(40,3 \%)$ y el apoyo de la familia $(31,7)$. Tener una casa grande fue mencionada por el 10\% de los encuestados.

Profundizando en las entrevistas, la preocupación económica futura (aunque también presente) más evidente fue la pensión de vejez. Muchos de ellos han proyectado las modificaciones y la construcción de su casa como una forma de compensar la inminente ausencia de pensión; así, Blanca, en Atenas, a quien ya se mencionó antes: "Ese apartamentico del tercero [piso] se hizo para arrendar. Lo construí, como para tener un tipo de pensión" (Blanca, entrevista personal, 5 de mayo, 2016). Mercedes (60 años) tiene en su vivienda una droguería y vive en el barrio Perdomo hace 40 años. Después de enviudar, y tras muchas gestiones y cursos, instaló la droguería, con el objetivo de que en su vejez no tuviera que depender de sus hijos: "porque hace 10 años se murió mi marido y no quería depender de mis hijos, es que yo vi tantos ejemplos de madres o padres que quedan solos y sufren mucho porque sus hijos no les colaboran" (Mercedes, entrevista personal, 27 de abril, 2016). 
A continuación, el relato de la esposa de César, del barrio San Martín de Porres, quien tienen una casa muy grande, en la cual arrienda 14 apartamentos y un local. Aunque contundente, preocupa su tono de desesperanza.

Este apartamento lo pensamos ya para morir acá. Dijo César: yo necesito un apartamento grande, para que si acaso, Dios no lo quiera, yo ya no pueda ni pararme y me pueda movilizar en una silla de ruedas. Esto se pensó así, para una pensión, porque uno viejo de dónde va a recibir un peso. Cuando se acabe de pagar esto, la casa nos va a dar unos 7 millones libres. Pero el problema es que ya uno no va a poder ni tomar una gaseosa, entonces ¿cuál es la gracia?, ya estamos muy agotados físicamente. Pero igual es la ilusión que tenemos. (Entrevista personal, 30 de abril, 2016)

La estrategia de movilidad social por excelencia es la apuesta por la educación de los hijos; la mayoría de los entrevistados lo mencionó como "la mejor herencia que uno les puede dejar". Pues bien, la vivienda sirvió de palanca en algunos casos, pero en otros fue más una barrera. Un ejemplo de lo primero es el caso de Bernarda, quien tiene una casa grande en el barrio Japón. Logró pagar en parte, los estudios universitarios de sus hijos gracias a los recursos que lograba obtener con la casa. Una de sus hijas es antropóloga y vive en un barrio central, su hijo es oficial del ejército y ha tenido mucho éxito en su carrera militar (entrevista personal, 7 de abril, 2016).

Todas las hijas de Obdulio, quien vive en el barrio Perdomo, son profesionales: "los recursos de la casa los hemos utilizado para ayuda de las hijas, para sacarlas adelante y que sean profesionales" (Obdulio, entrevista personal, 30 de abril, 2016). Lucio, de quien se habló antes y tiene una panadería en el barrio Atenas, permitió a sus hijos vivir en su casa con sus propios hogares para que no abandonaran sus estudios; incluso, apoyó el pago de la matrícula en universidades privadas (entrevista personal, 4 de mayo, 2016).

No obstante, se identificaron también casos de desinversión en el estudio de los hijos para favorecer la inversión en la vivienda. Sandra, una de las hijas de propietarios entrevistados en el barrio El anhelo, contaba, con frustración, como su madre había interrumpido estudios para construir la casa:

Cuando ella estaba construyendo la casa, era muy difícil conseguir cupo en un colegio distrital, y como ella no tenía para pagarnos estudio a las dos, le pagó a Pilar [su hermana]. Me dijo que no podía darme estudio hasta que no terminara la casa (...) y fue muy duro, porque tenía muchas ganas de estudiar (Sandra, entrevista personal, 20 de mayo, 2016) 
Si bien la estrategia de corto plazo fue priorizar la inversión en vivienda, la desinversión en educación puede tener efectos perversos en la movilidad social de los hijos a largo plazo.

\section{Conclusiones y discusión}

La construcción de la vivienda, en los sectores populares, es un proceso de toda la vida, y requiere una compleja red de decisiones y acciones para llegar al resultado actual: Viviendas grandes, de dos o tres pisos, subdivididas en apartamentos y con espacios productivos habitadas por varios hogares, por una y hasta tres generaciones.

La edad del jefe de hogar y su posición en el ciclo de vida implican una condición de vulnerabilidad económica, en la cual su vivienda adquiere relevancia como estrategia de sobrevivencia cotidiana. La mayoría de propietarios utilizan su vivienda para obtener ingresos y en muchos casos constituye el principal o el único ingreso de la familia.

En cuanto a las crisis económicas, aunque se prioriza incrementar la fuerza laboral y disminuir el gasto, la casa es un recurso cada vez más relevante para contenerlas. Lo anterior explica que en momentos de bonanza la primera opción sea invertir en su mejora y ampliación.

Y es que la vivienda es considerada, en sí misma, como una estrategia de movilidad social.
Permite aumentar el patrimonio a futuro y contener la carencia de pensión; y en perspectiva intergeneracional, permite apalancar la educación superior de los hijos, aunque también su construcción implique, a veces, una desinversión en capital humano, afectando las posibilidades de movilidad social en el largo plazo.

Para un diálogo de los resultados con la teoría, es preciso retomar lo planteado por Wallace (2002), en cuanto a ver el enfoque en sus tres dimensiones: 1) como concepto, lo que permite visibilizar la capacidad de agencia de los hogares en su adaptación a los cambios; 2) como método, en el análisis de la relación de las estrategias con la estructura social; y 3) como unidad de análisis, en términos de la centralidad del hogar.

Para aportar al enfoque de las EFV en cuanto concepto, en términos de las estrategias y la capacidad de agencia de los hogares, los resultados confirman la enorme importancia económica de la vivienda propia para estos sectores, así como su capacidad para movilizarla. Y es que la vivienda aumenta la resiliencia de los hogares vulnerables hasta el límite de su creatividad.

Sin embargo, en cuanto a la relación de tales estrategias con la estructura social, las múltiples funciones sociales y económicas que asume la vivienda (reemplazar la pensión de vejez, contener crisis por desempleo o enfermedad, solventar la escasez de oferta de vivienda para los jóvenes) contribuyen a ocultar y reproducir las enormes carencias en la oferta de seguridad 
social. Las estrategias de vida cobran más sentido en la medida en que el Estado delega a los ciudadanos y al sector privado el bienestar de la población, lo que se evidencia claramente en los casos analizados (Wallace, 2002). Los efectos de estas ausencias en la seguridad social no son homogéneos en la sociedad, por lo que la posición del hogar en la estructura social adquiere relevancia. Estos hogares han tenido que afrontar las carencias de la provisión social desde su formación: la misma auto-construcción de la vivienda, la gestión colectiva de los servicios urbanos, su paulatina dotación urbana. Y actualmente las siguen padeciendo, aunque más silenciosamente. La resiliencia de los hogares, paradójicamente, pareciera reforzar los mecanismos reproductores de la desigualdad.
Finalmente, y en términos de la unidad de análisis, los resultados de este estudio plantean una reflexión sobre la relación entre el hogar y la vivienda, apareciendo esta última en el estudio como un recurso que los hogares pueden movilizar para protegerse ante los cambios y disminuir su vulnerabilidad, e incluso, para promover un proceso de movilidad social. La vivienda comporta, entonces, múltiples sentidos en relación con el hogar y el habitar, y si bien mantiene un enorme valor de uso, refuerza también su papel en la reproducción individual, del hogar, de la fuerza de trabajo y del capital (Rugiero Pérez, 2009). 


\section{Referencias bibliográficas}

Álvarez, M. (2009). Informalidades: efectos de la informalidad urbana sobre el empleo de los jóvenes. Política y Gestión, (11).

Arteaga, C. (2007). Pobreza y estrategias familiares: debates y reflexiones. Revista Mad, (17), 144164. doi:10.5354/0718-0527.2011.13942

Aulestia, D. (2009). Medios de vida urbanos y vivienda En Intersecciones urbanas: origen y contexto en América Latina. Quito: FLACSO.

Barabino, N., Bocero, S., Prandín, G., y Rosenthal, C. (s.f.). Estrategias de sobrevivencia, racionalidad y reproducción social. Observatorio Geográfico de América Latina. http://observatoriogeograficoamericalatina.org.mx/egal6/Geografiasocioeconomica/Geografiaeconomica/427.pdf

Bazán, L. (1999). Casa y familia. Los recursos de los desempleados de Pemex en la ciudad de México. Estudios Sociológicos, 17(50), 473-498.

Bericat, E. (1998). La integración de los métodos cuantitativo y cualitativo en la investigación social. Significado y medida. Barcelona: Ariel.

Bourdieu, P. y Lamaison, P. (1985). De la règle aux stratégies: entretien avec P. Bourdieu. Terrains, (4), 93-101. doi:10.4000/terrain.2875

Bourdieu, P. (1988). La distinción. Criterio y bases sociales del gusto. Buenos Aires: Taurus.
Bourdieu, P. (1989). La ilusión biográfica. Historia y Fuente Oral, (2), 27-33. Recuperado de www. jstor.org/stable/27753247

Bourdieu, P. (2011). Las estrategias de reproducción social. Buenos Aires: Siglo Veintiuno.

Camargo, A. (2014). New approaches to intervention in the informally settled areas of Bogotá. En P. Ward, E. Jiménez, y M. di Virgilio, Housing policy in Latin American cities: A new generation of strategies and approaches for 2016 UN-HABITAT III. New York: Routledge.

Camargo, A. y Hurtado, A. (2013). Urbanización informal en Bogotá: agentes y lógicas de producción del espacio urbano. Revista INVI, 28(78), 77-107. doi:10.4067/ S0718-83582013000200003

Cornia, A. (1987). Adjustement at the household level: potentials and limitations or survival strategies. En G. Cornia, R. Jolly, y F. Stewart (Eds.), Adjustment with a human face. Volume 1: Protecting the vulnerable and promoting growth. Oxford: Claredon Press.

Di Virgilio, M. (2011). La movilidad residencial: una preocupación sociológica. Territorios, (25), 173-190

Duque, J. y Pastrana, E. (1973). Las estrategias de supervivencia económica de las unidades familiares del sector popular urbano: una investigación exploratoria. Santiago de Chile: ELAS-CELADE. 
Eguía, A. (2004) Pobreza y reproducción familiar: propuesta de un enfoque para su estudio. Caderno CRH, 17(40), 79-92. doi:10.9771/ccrh. v17i40.18481

Elder, G. (2000). The life course. En E. F. Borgatta y R. J. V. Montgomery (Eds.), Encyclopedia of sociology (2a ed., vol. 3, pp. 1614-1622). New York: Macmillan.

Gaviria, A. (2005). Movilidad social en Colombia: realidades y percepciones. Bogotá: Departamento Nacional de Planeación.

Garrido, L. y Gil, E. (1996). El concepto de estrategias familiares. En L. Garrido y E. Gil (Eds.), Estrategias familiares. Madrid: Alianza.

Giddens, A. (1995) La constitución de la sociedad: bases para la teoría de la estructuración. Buenos Aires: Amorrortu.

González de la Rocha, M. (1986). Los recursos de la pobreza. Familias de bajos ingresos de Guadalajara. Guadalajara: El Colegio de Jalisco, CIESAS/ SPP.

González de la Rocha, M. (2009). Procesos domésticos y vulnerabilidad, perspectivas antropológicas de los hogares con oportunidades. México: CIESAS.

Gutiérrez, A. (2005). Pobre como siempre. Estrategias de reproducción social en la pobreza. Córdoba: Eduvim.

Hintze, S. (2004). Capital social y estrategias de supervivencia. Reflexiones sobre el «capital social de los pobres». En C. Danani (Comp.),
Politicas sociales y economía social: debates fundamentales. Buenos Aires: UNGS.

Jiménez, E. y Camargo, A. (2015). Rental markets and housing policies in consolidated informal settlements. En P. Ward, E. Jiménez y M. di Virgilio. Housing policy in Latin American cities: a new generation of strategies and approaches for 2016 UN-habitat III. New York: Routledge.

Katzman, R. (1999). Activos y estructuras de oportunidades: estudios sobre las raices de la vulnerabilidad social en Uruguay. Montevideo: CEPAL.

Moser, C. (1996). How do the urban poor manage in an economic crisis? Finance and development, 33(4), 42-44.

Moser, C. (2009). Gente del barrio, vidas extraordinarias. Activos y reducción de la pobreza en Guayaquil 1978-2004. Santiago de Chile: Ediciones Sur.

Moen, P. y Wethington, E. (1992). The concept of family adaptative strategies. Annual Review of Sociology, 18, pp. 233-251. doi:10.1146/annurev.so.18.080192.001313

Núñez, J. (2012). Pobreza, empleo y movilidad social: evidencia e interpretación de los problemas sociales en Colombia. (Tesis Doctorado en Ciencias Sociales y Humanas, Pontificia Universidad Javeriana, Bogotá, Colombia).

Ortiz, N. y Diaz, C. (2018). Una mirada a la vulnerabilidad social desde las familias. Revista Mexicana de Sociología, 80(3), 611-638. doi:10.22201/iis.01882503p.2018.3.57739 
Peattie, L. (1974). The concept of marginality as applied to squatter settlements. En W. A. Cornelius y F. M. Trueblood, (Eds.), Latin American Urban Research (vol. 4, pp. 101-109). California: Sage.

Perlman, J. (2010). Favela. Four decades of living on the edge in Rio de Janeiro. Oxford: Oxford University Press.

Prada, M. F. (2006). Los hogares colombianos ante los choques: efectividad de los mecanismos de protección social (Documento CEDE 200623). Recuperado de https://ideas.repec.org/p/ $\mathrm{col} / 000089 / 003170 . \mathrm{html}$

Rugiero Pérez, A. (2009). Aspectos teóricos de la vivienda en relación al habitar. Revista INVI, 15(40), 67-97.

Stake, R. (1994). Case studies. En N. K. Denzin e Y. Lincoln (Eds.), Handbook of Qualitative Methods (2a ed.). Thousand Oaks: Sage.

Sautú, R. (1999). El método biográfico: la reconstrucción de la sociedad a partir del testimonio de los actores. Buenos Aires: Editorial de Belgrano.

Sáenz, Á. y Di Paula, J. (1981). Precisiones teóricometodológicas sobre la noción de estrategias de existencia. Demografía y Economía, 15(2), 149-163. doi:10.24201/edu.v15i02.509

Torrado, S. (1982). El enfoque de las estrategias familiares de vida en América Latina. Orientaciones teórico metodológicas. Argentina: CEUR.
Vallejos, E. y Leotta, D. (2013). Apuntes teóricos y metodológicos sobre el concepto de estrategias familiares de vida. Avances de investigación sobre un estudio de caso. En X Jornadas de Sociología. Facultad de Ciencias Sociales, Universidad de Buenos Aires, Buenos Aires.

Wallace, C. (2002). Household strategies: Their conceptual relevance and analytical cope in social research. Sociology, 32(2), 275-292. doi:10.1177/0038038502036002003

Ward, P. M., Jiménez, E., y Di Virgilio, M. (2014). Intensive case study methodology for the analysis of self-help housing consolidation, household organization and family mobility. $\mathrm{Cu}$ rrent Urban Studies, 2(2), 88-104. doi:10.4236/ cus. 2014.22010

Ward, P. M., Jiménez, E., Di Virgilio, M., y Camargo, A. (2015). Politicas de vivienda en ciudades latinoaméricanas: una nueva generación de estrategias y enfoques para 2016 ONU-Habitat III. Bogotá: Universidad del Rosario.

Zaffaroni, C. (1999). Los recursos de las familias urbanas de bajos ingresos para enfrentar situaciones críticas. En Katzman, R. (Coord.), Activos y estructura de oportunidades. Estudio sobre las raíces de la vulnerabilidad social en Uruguay. Montevideo: Cepal. 


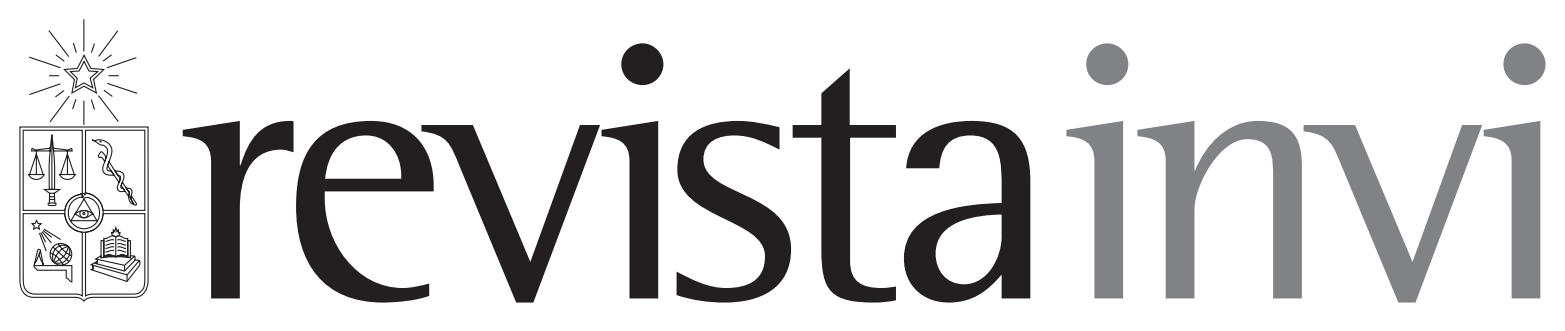

Revista INVI es una publicación periódica, editada por el Instituto de la Vivienda de la Facultad de Arquitectura y Urbanismo de la Universidad de Chile, creada en 1986 con el nombre de Boletín INVI. Es una revista académica con cobertura internacional que difunde los avances en el conocimiento sobre la vivienda, el hábitat residencial, los modos de vida y los estudios territoriales. Revista INVI publica contribuciones originales en español, inglés y portugués, privilegiando aquellas que proponen enfoques inter y multidisciplinares y que son resultado de investigaciones con financiamiento y patrocinio institucional. Se busca, con ello, contribuir al desarrollo del conocimiento científico sobre la vivienda, el hábitat y el territorio y aportar al debate público con publicaciones del más alto nivel académico.

Director: Dr. Ricardo Tapia Zarricueta, Universidad de Chile, Chile.

Editor: Dr. Luis Campos Medina, Universidad de Chile, Chile.

Editor asistente: Dr. Walter Imilan, Universidad de Chile, Chile.

Coeditora: Srta. Sandra Rivera, Universidad de Chile, Chile.

\section{COMITÉ EDITORIAL:}

Dr. Victor Delgadillo, Universidad Autónoma de la Ciudad de México, México.

Dra. María Mercedes Di Virgilio, CONICET/ IIGG, Universidad de Buenos Aires, Argentina.

Dra. Irene Molina, Uppsala Universitet, Suecia.

Dr. Gonzalo Lautaro Ojeda Ledesma, Universidad de Valparaíso, Chile.

Dra. Suzana Pasternak, Universidade de São Paulo, Brasil.

Dr. Javier Ruiz Sánchez, Universidad Politécnica de Madrid, España.

Dra. Elke Schlack Fuhrmann, Pontificia Universidad Católica de Chile, Chile.

Dr. Carlos Alberto Torres Tovar, Universidad Nacional de Colombia, Colombia.

Sitio web: http://www.revistainvi.uchile.cl/

Correo electrónico: revistainvi@uchilefau.cl

Licencia de este artículo: Creative Commons Atribución-Compartirlgual 4.0 Internacional (CC BY-SA 4.0) 